\title{
Generalized Maximum-Likelihood Sequence Detection for Photon-Counting Free Space Optical Systems
}

\author{
Nestor D. Chatzidiamantis, Student Member, IEEE, George K. Karagiannidis, Senior Member, IEEE, \\ and Murat Uysal, Senior Member, IEEE
}

\begin{abstract}
We investigate detection methods for on-off keying (OOK) photon-counting Free Space Optical (FSO) systems in the presence of turbulence-induced fading, assuming no channel state information at the receiver. To recover the performance loss which is associated with symbol-by-symbol detection in such a scenario, we consider sequence detection techniques, exploiting the temporal correlation of the FSO channel. Due to its high complexity in the calculation of its metric, optimal maximum likelihood sequence detection (MLSD) is infeasible for most practical purposes. Hence, we propose a suboptimal low-complexity detection rule, which is based on the generalized maximum-likelihood sequence estimation. The proposed scheme allows the detection of sequence lengths that are prohibitive for conventional MLSD, without using any kind of channel knowledge. Monte Carlo simulation results show its performance to be very close to the optimum for large sequence lengths and various fading models.
\end{abstract}

Index Terms-Free-space optical systems, turbulence-induced fading, poisson photon counting model, maximum likelihood sequence detection, generalized maximum likelihood sequence detection.

\section{INTRODUCTION}

$\mathbf{F}$ REE-SPACE optical (FSO) communication is a wireless technology which has recently attracted much interest within the research community, due to its potential use in various applications [1]. However, despite their significant advantages, the widespread deployment of FSO systems is limited by their high vulnerability to adverse atmospheric conditions. Even in a clear sky, due to inhomogeneities in temperature and pressure changes, the refractive index of the atmosphere varies and results in atmospheric turbulence. This causes rapid fluctuations at the intensity of the received optical signal, known as turbulence-induced fading, that severely affect the overall reliability of the FSO link.

Most commercial FSO systems employ on-off keying (OOK) intensity modulation (IM) and direct detection (DD), as transmission and reception mechanisms. Hence, detection methods for such systems have been investigated by several authors. Detection with perfect channel state information (CSI), i.e. the receiver has perfect knowledge of the channel's instantaneous fading intensity, has been theoretically studied

Paper approved by J. Liu, the Editor for Free Space Optics and Hybrid RF/Optical Wireless Systems of the IEEE Communications Society. Manuscript received July 21, 2009; revised December 8, 2009.

N. D. Chatzidiamantis and G. K. Karagiannidis are with the Wireless Communications Systems Group (WCSG), Department of Electrical and Computer Engineering, Aristotle University of Thessaloniki, GR-54124 Thessaloniki, Greece (e-mail: \{nestoras, geokarag\} @auth.gr).

M. Uysal is with the School of Engineering, Özyeğin University, 34662, Altunizade, Uskudar, Istanbul, Turkey (e-mail: murat.uysal@ozyegin.edu.tr).

Digital Object Identifier 10.1109/TCOMM.2010.093010.090116A in [2]; however, its practical implementation requires pilotsymbol assisted receiver structures [3], which result in the transmission of redundant overhead and increased implementation complexity (need of data framing and packetization [4]). As a consequence, detection techniques with no CSI have attracted the attention. In [5], a simple symbol-by-symbol maximum-likelihood (ML) detector has been proposed, which used the channel's statistics for determining the threshold in the detection process of the OOK bits; nevertheless, this detector suffers from significant performance loss.Hence, in the same work, the maximum likelihood sequence detector (MLSD) has been proposed as an alternative, which performs joint detection of multiple symbols based on the channel's temporal and statistical characteristics. Despite its efficiency, MLSD suffers from high complexity, since it involves the computation of complicated integrals in its metric. Although low complexity MLSD solutions have been suggested in [4] and [6] for different detection models, the requirement for knowledge of the channel's fading statistical characteristics still makes the implementation of this method impractical. In order to overcome these difficulties, a blind detection technique has been proposed in [7], where no channel knowledge is available at the receiver. The performance of this technique turns out to be very close to the perfect CSI receiver; however, its application is restricted to backgroundnoise limited receivers, which build upon the approximating Gaussian detection model.

In this paper, we investigate blind detection techniques for OOK FSO systems, which are described by the more general Poisson photon-counting detection model. Based on the generalized ML sequence estimation (GMLSE) principle, introduced in [8] and [9], we extend the work of [7] and propose a simple sequence detection rule, which exploits efficiently the temporal characteristics of turbulence induced fading. Using only the observed values of the received sequence, the new detection rule avoids the calculation of complicated decision metrics and does not require any kind of channel knowledge. The performance of the proposed detector is investigated for different sequence lengths and compared with the conventional MLSD and perfect CSI receiver. We further provide simulation results that illustrate the robustness of the proposed receiver structure, irrespective the assumed turbulence conditions and fading models.

The remainder of the paper is organized as follows. In section II, we describe the turbulence-induced fading models of interest and the system model. In section III, we present the perfect CSI and MLSD detectors, which will serve as benchmarks, and introduce the generalized ML sequence detection 
method. In section IV, we present Monte-Carlo simulation results for the error rate performance of the proposed detection technique and compare it with benchmarking schemes. Section $\mathrm{V}$ concludes the paper.

\section{SySTEM ModeL}

We consider an FSO communication system that employs IM/DD and OOK modulation, operating over atmospheric turbulence induced fading channel.

\section{A. Atmospheric Turbulence Models}

Atmospheric turbulence is a major performance-degrading factor in FSO systems, which leads to intensity variations of the received optical signals. For its statistical description, various statistical models have been proposed in the literature [1], depending on the turbulence strength. The scintillation index, defined as [1]

$$
\text { S.I. } \triangleq \frac{E\left\{I^{2}\right\}}{(E\{I\})^{2}}-1
$$

where $I$ represents the intensity of the received optical signals, is a measure of the turbulence strength. Lower values of scintillation index lead to less intensity variations, while higher values result in more severe turbulence-induced fading. Moreover, without loss of generality, it is assumed that the mean of the intensity variations is normalized, i.e. $E\{I\}=1$.

In weak turbulence conditions, the most widely accepted fading model is lognormal (LN). In this case, the logarithm of the intensity variations is normally distributed and the probability density function (PDF) of $I$ is given by

$$
p_{I}(I)=\frac{1}{I \sqrt{2 \pi \sigma_{x}^{2}}} \exp \left\{-\frac{\left(\ln (I)-m_{x}\right)^{2}}{2 \sigma_{x}^{2}}\right\}
$$

where $m_{x}=-0.5 \ln ($ S.I. +1$)$ and $\sigma_{x}^{2}=\ln ($ S.I. +1$)$.

In moderate to strong turbulence conditions, the recently proposed Gamma-Gamma (GG) model can be used for the statistical description of turbulence-induced fading. According to this model, intensity fluctuations are considered to be derived from the product of small-scale and large-scale fluctuations, both statistically defined by Gamma distribution [1]. Hence, in this case, the PDF of the intensity variations is given by

$$
p_{I}(I)=\frac{2(\alpha \beta)^{\frac{\alpha+\beta}{2}}}{\Gamma(\alpha) \Gamma(\beta)} I^{\frac{\alpha+\beta-2}{2}} K_{a-\beta}(2 \sqrt{\alpha \beta I})
$$

where $\Gamma(\cdot)$ is the gamma function [10, Eq. (8.310)], $K_{\nu}(\cdot)$ is the $\nu$ th order modified Bessel function of the second kind [10, Eq. (8.432/9)], and $\alpha$ and $\beta$ are the parameters which are related with effective atmospheric conditions [1]. The scintillation index is calculated according to S.I. $=\alpha^{-1}+$ $\beta^{-1}+(\alpha \beta)^{-1}$.

Lastly, as the strength of the turbulence increases, the statistics of turbulence-induced fading can be modeled using the negative exponential distribution, which is considered as the limit distribution approached when the number of scatterers is large. In this case, the PDF of intensity variations is given by

$$
p_{I}(I)=\exp \{-I\}
$$

and the scintillation index is equal to unity.

\section{B. Signal Model}

Let $s[k] \in\{0,1\}$ denote the OOK transmitted signal corresponding to the $k$ th symbol interval. At the receiver end of the FSO system, the optical field which is collected from the receive aperture, is focused on a photodetector (PD). Similar to [6] and [11], we adopt the Poisson photon-detection model, where the output of the photodetector can be modeled as a Poisson point process whose rate is proportional to the total optical power incident on it ${ }^{1}$.

The detected signal at the receive PD during the $k$ th symbol interval, $r[k]$, is a Poisson count variable with probability mass function (PMF) expressed as [6]

$$
\begin{aligned}
\operatorname{Pr}\{r[k] \mid s[k], I[k]\}= & \frac{\left(n_{s} s[k] I[k]+n_{b}\right)^{r[k]}}{r[k] !} \times \\
& \exp \left\{-\left(n_{s} s[k] I[k]+n_{b}\right)\right\}(5)
\end{aligned}
$$

where $n_{s}=\eta P_{s} T /(h f)$ is the effective count parameter due to the transmitted power and $n_{b}=\eta P_{b} T /(h f)$ is the effective count parameter due to the background radiation. Here, $\eta$ corresponds to the receiver's optical-to-electrical efficiency, $T$ is the duration of a bit interval, $f$ is the center frequency of the transmission, $h=6.6 \times 10^{-34} \mathrm{~J} \cdot s$ is Planck's constant, $P_{s}$ is the total transmitted optical power and $P_{b}$ is the power incident on the PD from background noise. Furthermore, the term $I[k]$ denotes the turbulence-induced fading coefficient that corresponds to the $k$ th bit interval.

It must be noted that atmospheric turbulence results in a very slowly-varying fading in FSO systems. For the signalling rates of interest ranging from hundreds to thousands of Mbps [13], turbulence-induced fading can be considered as constant over hundred of thousand or millions of consecutive symbols, since the coherence time of the channel is about $1-100 \mathrm{~ms}$ [14]. Hence, it is assumed that $I[k]$ in (5) remains constant over an observation window of $K$ symbols and therefore we drop the time index $k$, i.e.

$$
I=I[k], k=1, \ldots K .
$$

The signal-to-noise ratio (SNR) is defined as the ratio of the squared expected mean of the information bearing component to the total variance of the received signal [6], [15]. Hence, according to (5), SNR is defined as

$$
\gamma=\frac{\left(n_{s} \mu_{I}\right)^{2}}{2 n_{s} \mu_{I}+4 n_{b}}
$$

where $\mu_{I}=E\{I\}=1$.

\section{Sequence Detection Techniques}

In this section, we present sequence detection techniques which are based on the ML criterion and can be employed when CSI is not available at the receiver. For the convenience of the reader, the receiver which has perfect knowledge of the instantaneous CSI will also be described, since its performance constitutes a lower bound (genie bound) to the performance of the receivers operating without CSI.

\footnotetext{
${ }^{1}$ Note that the approximating Gaussian detection model which has been employed in [5] and [12] can be adopted only in the special case where high signal energies are considered.
} 


\section{A. Receiver with Perfect CSI}

Given the received signal $r[k]$ and the channel's instant fading state $I$, the decision rule of the receiver with perfect CSI will be

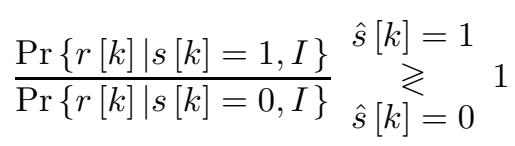

which according to (5) reduces to

$$
\left(\frac{n_{s}}{n_{b}} I+1\right)^{r[k]} \exp \left\{-n_{s} I\right\} \stackrel{\hat{s}[k]=1}{\underset{s}{\gtrless}[k]=0} 1
$$

\section{B. $M L S D$}

This detection scheme exploits the temporal correlation of turbulence-induced fading over consecutive transmitted symbols. MLSD has been earlier investigated for Poisson detection model in [6] and performs joint detection of the transmited sequence, $\mathbf{s}=\{s[k]\}_{k=1}^{K}$, based on the decision rule given by

$$
\begin{aligned}
\hat{\mathbf{s}} & =\arg \max _{\mathbf{s}} \prod_{k=1}^{K} \operatorname{Pr}\{r[k] \mid s[k]\} \\
& =\arg \max _{\mathbf{s}} \int_{0}^{\infty} \prod_{k=1}^{K} \operatorname{Pr}\{r[k] \mid s[k], I\} p_{I}(I) d I(10)
\end{aligned}
$$

where it is assumed that turbulence-induced fading remains constant over the sequence of $K$ consecutive transmitted symbols and $\hat{\mathbf{s}}=\{\hat{s}[k]\}_{k=1}^{K}$ is the estimated transmitted sequence. This decision rule can be further rewritten as [6]

$$
\hat{\mathbf{s}}=\arg \max _{\mathbf{s}} \int_{0}^{\infty}\left(\frac{n_{s}}{n_{b}} I+1\right)^{R_{o n}} \exp \left\{-n_{s} N_{o n} I\right\} p_{I}(I) d I
$$

where $N_{\text {on }} \in\{0,1, \ldots, K\}$ is defined to be the number of ones in the hypothesis vector,

$$
R_{o n} \triangleq \sum_{k_{i} \in S_{o n}} r\left[k_{i}\right]
$$

is the sum of the received signals that correspond to the indices of the ones in the hypothesis vector, and $S_{\text {on }} \triangleq$ $\left\{k_{i} \in\{1,2, \ldots, K\}: s\left[k_{i}\right]=1\right\}$ is the size- $N_{\text {on }}$ of on-indices.

A major drawback of MLSD is the computational complexity of its metric. This complexity stems from the fact that the calculation of the MLSD metric, according to (11), cannot be performed via analytical closed-form expressions, due to the mathematically intricate nature of the fading distributions. Moreover it requires knowledge of the channel's fading statistics and hence its performance depends on the assumed turbulence conditions. A suboptimal MLSD metric, which offers analytical tractability through the approximation of the fading distribution, has been proposed in [6]; however it still requires knowledge of the channel's fading statistics.

\section{Generalized MLSD}

In this subsection, we propose a new detection method, based on the GMLSE principle [8]-[9], as an alternative to the original MLSD. Generalized maximum likelihood sequence detection (GMLSD) is in general a two-step process which estimates both the transmitted sequence and the channel state using only the observation window of consecutive received symbols.

GMLSD is based on the decision rule

$$
\hat{\mathbf{s}}=\arg \max _{\mathbf{s}} l\{\mathbf{r} \mid \mathbf{s}, \hat{I}\}
$$

where $\hat{I}$ is an estimate of the fading coefficient calculated through

$$
\hat{I}=\arg \max _{I} l\{\mathbf{r} \mid \mathbf{s}, I\}
$$

and the conditioned on $I$ maximum-likelihood function, given by [6]

$$
l\{\mathbf{r} \mid \mathbf{s}, I\}=\prod_{k=1}^{K} \operatorname{Pr}\{r[k] \mid s[k], I\} .
$$

According to (5), (15) can be expressed as

$$
l\{\mathbf{r} \mid \mathbf{s}, I\}=\prod_{k=1}^{K} \frac{\left(n_{s} s[k] I+n_{b}\right)^{r[k]}}{r[k] !} \exp \left\{-\left(n_{s} s[k] I+n_{b}\right)\right\}
$$

After eliminating irrelevant terms, the conditioned maximumlikelihood function in (16) can be modified to

$$
l\{\mathbf{r} \mid \mathbf{s}, I\}=\left(\frac{n_{s}}{n_{b}} I+1\right)^{R_{o n}} \exp \left\{-n_{s} N_{o n} I\right\}
$$

where $N_{o n}$ and $R_{o n}$ are earlier defined.

By taking the derivative of (17) with respect to $I$ and setting it to equal to zero, the fading intensity that maximizes (14) is obtained as

$$
\hat{I}=\frac{1}{n_{s}}\left(\frac{R_{o n}}{N_{o n}}-n_{b}\right) .
$$

Hence, by inserting the channel's estimate in (17), the decision rule of GMLSD becomes

$$
\hat{\mathbf{s}}=\arg \max _{\mathbf{s}}\left(\frac{R_{o n}}{N_{o n}} \frac{1}{n_{b}}\right)^{R_{o n}} \exp \left\{-R_{o n}+n_{b} N_{o n}\right\} .
$$

It is evident from (19) that GMLSD decision rule:

- avoids the calculation of the complex integral of the MLSD metric and its decision is based solely on the observed values of received sequence.

- does not require any knowledge of the fading distribution, and, hence, it can be employed in various turbulence conditions.

In practical implementation, GMLSD requires at least one symbol in the transmitted sequence $\mathbf{s}$ to correspond to the onstate. Similarly to the blind detection method of [7], when the all off-state sequence is transmitted, the detection process will exhibit errors, even as SNR approaches infinity, due to the estimation ambiguity. Hence, taking into consideration that all transmitted sequences are equally likely, the bit error performance (BER) of the proposed detection method is limited by an error floor, whose exact value ranges between

$$
\frac{1}{K} \operatorname{Pr}\left\{N_{\text {on }}=0\right\}<B E R_{\text {floor }}<\operatorname{Pr}\left\{N_{\text {on }}=0\right\}
$$

where $\operatorname{Pr}\left(N_{\text {on }}=0\right)=\frac{1}{2^{K}}$. Since the error floor reduces with increasing $K$, GMLSD can be considered particularly effective for large sequence lengths, i.e. large number of consecutive symbols where the fading remains constant, which is easily justified for the slowly varying atmospheric turbulence channels. 


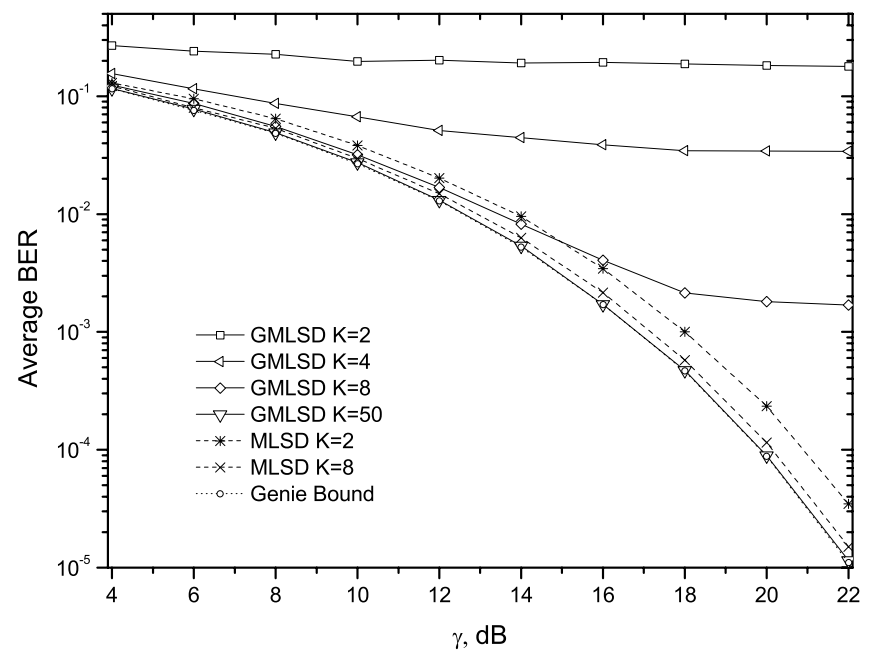

Fig. 1. Comparison of GMLSD and MLSD for different sequence lengths, when weak turbulence conditions and LN fading model are assumed (S.I. = $0.5)$.

\section{Simulations Results and Discussion}

In this section, we present Monte-Carlo simulation results for the BER performance of the GMLSD receiver in various turbulence conditions. We further compare its performance with the benchmarking schemes of MLSD receiver and the perfect CSI receiver. Adopting previously reported link parameters [6], the photodetector efficiency is set to $\eta=0.5$, the optical frequency is assumed to be $f=1.94 \times 10^{14} \mathrm{~Hz}$ and let $P_{b} T=-170 \mathrm{~dB}$, which corresponds to an average of $n_{b}=39$ background radiation photoelectrons per bit interval. Moreover, the fast search algorithm developed in [4] is employed for searching the maximum metrics of both MLSD and GMLSD methods over all the possible transmitted sequences.

In Fig. 1, we consider a FSO communication system over weak turbulence conditions and LN fading model. As it is clearly depicted in the figure, the BER performance of the GMLSD receiver is limited from the probability of transmitting the all-off sequence. However, in accordance with (20), the error floor reduces as the sequence length $K$ increases and diminishes for large sequence lengths, such as $K=50$. Compared with MLSD receiver structure of the same sequence length, the simulation results indicate that MLSD clearly outperforms GMLSD, especially at the high SNR regime. However, the complexity of the MLSD increases significantly as the sequence length increases and hence there is a restriction on the maximum sequence length that can be simulated (in this paper we were able to simulate up to $K=8$ ). In contrast, large sequence lengths can be easily handled through GMLSD. For sufficiently large sequence lengths $(K=50)$, the performance of GMLSD becomes identical to the genie bound.

In Figs. 2 and 3, the performance of the FSO communication system under consideration is investigated over moderate ${ }^{2}$ and very strong turbulence conditions, respectively. It is ob-

\footnotetext{
${ }^{2}$ It should be noted that in the case of Gamma-Gamma fading model, the parameters $\alpha$ and $\beta$ are not chosen arbitrarily, but they are calculated based on the considered Rytov variance, $\sigma_{1}^{2}$, and the ratio of the inner scale, $l_{o}$, to the Fresnel zone, $R_{F}[1]$.
}

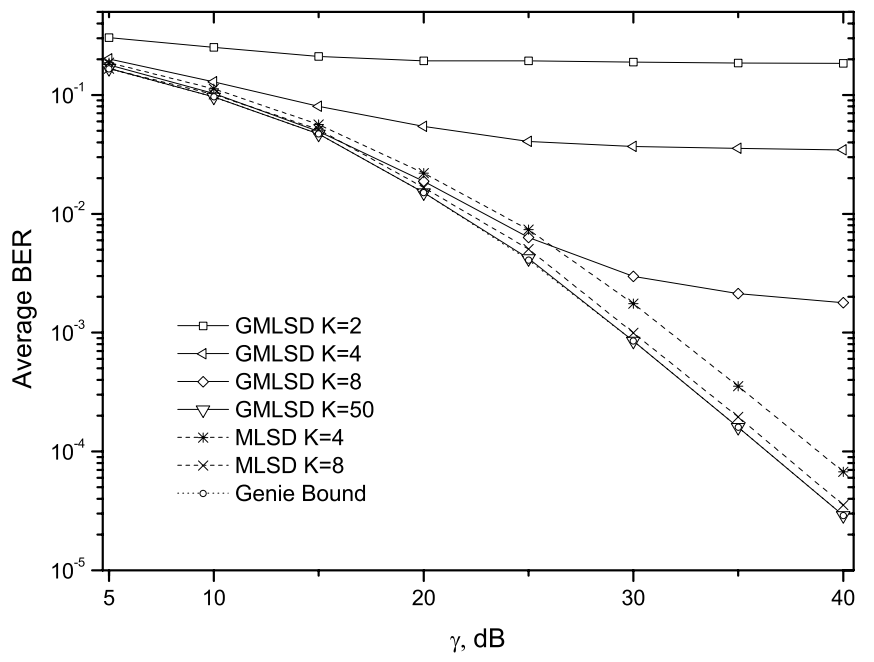

Fig. 2. Comparison of GMLSD and MLSD for different sequence lengths, when moderate turbulence conditions $\left(\sigma_{1}^{2}=2, \frac{l_{o}}{R_{F}}=0.5\right)$ and GammaGamma fading model are assumed (S.I. $=1.38, \alpha=2.23, \beta=1.54$ ).

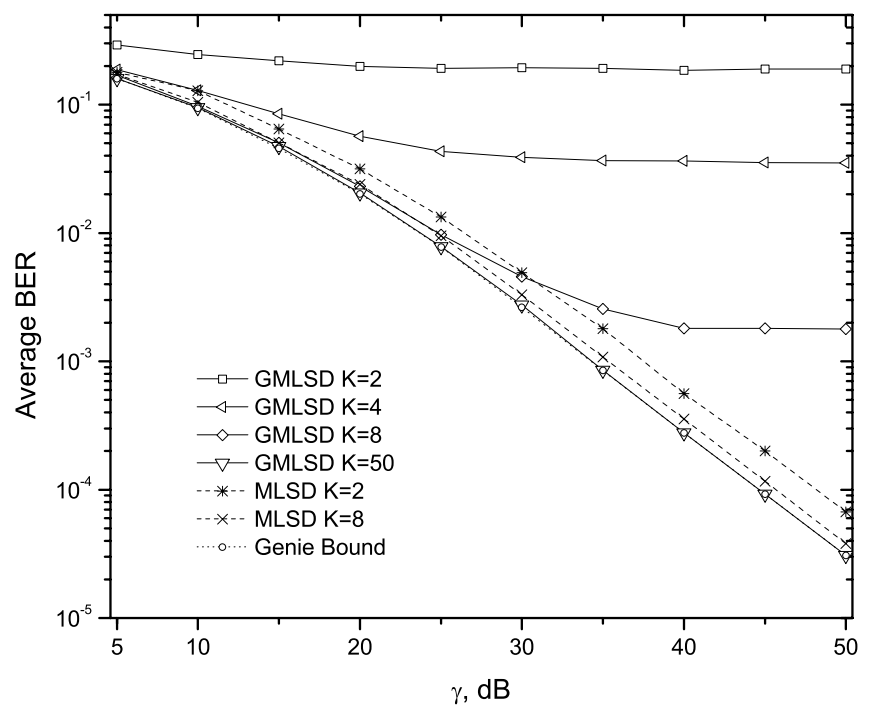

Fig. 3. Comparison of GMLSD and MLSD for different sequence lengths at the saturation of turbulence, where negative exponential fading model is assumed (S.I. $=1)$.

vious from both figures that the performance of the GMLSD technique depends only on the sequence length $K$, irrespective the turbulence strength or the statistics of the fading model. This was expected, since GMLSD decision rule does not take into consideration the statistics of the channel, but only the observation window of received optical signals. Moreover, as in weak turbulence conditions, MLSD always outperforms GMLSD for shorter sequences. However, GMLSD improves for larger sequence lengths that are prohibitive for MLSD and thus performance identical to the perfect CSI receiver is achieved.

\section{CONCLUSions}

We have investigated sequence detection techniques for FSO systems in the presence of turbulence-induced fading. Sequence detection exploits the temporal correlation of FSO channel and promises performance close to the genie bound. 
However, optimal MLSD is infeasible for most practical purposes due to its high complexity in the calculation of its metric. Moreover it requires knowledge of the channel's atmospheric conditions and fading model. In our work, we have proposed a suboptimal low-complexity ML sequence detector, which is based on the GMLSE principle, assuming the general Poisson photon-counting detection model. Although the proposed receiver structure performs poorly for small observation windows, its low complexity allows the joint detection of large sequences of consecutive transmitted symbols, achieving the genie bound performance.

\section{REFERENCES}

[1] L. Andrews, R. L. Philips, and C. Y. Hopen, Laser Beam Scintillation with Applications. SPIE Press, 2001.

[2] M. Uysal, S. M. Navidpour, and J. Li, "Error rate performance of coded free-space optical links over strong turbulence channels," IEEE Commun. Lett., vol. 8, no. 10, pp. 635-637, Oct. 2004.

[3] X. Zhu and J. M. Kahn, "Pilot-symbol assisted modulation for correlated turbulent free-space optical channels," in Proc. SPIE Intl. Symp. Optical Science Technol., San Diego, CA, 2001.

[4] M. L. B. Riediger, R. Schober, and L. Lampe, "Fast multiple-symbol detection for free-space optical communications," IEEE Trans. Commun., vol. 57, no. 4, pp. 1119-1128, Apr. 2009.

[5] X. Zhu and J. M. Kahn, "Free-space optical communication through atmospheric turbulence channels," IEEE Trans. Commun., vol. 50, no. 8, pp. 1293-1300, Aug. 2002.
[6] M. L. B. Riediger, R. Schober, and L. Lampe, "Multiple-symbol detection for photon-counting MIMO free-space optical communications," IEEE Trans. Wireless Commun., vol. 7, no. 12, pp. 5369-5378, Dec. 2008.

[7] — "Blind detection of on-off keying for free-space optical communications," in Proc. VCCECE/CCGEI, Niagara Falls, Canada, 2008, pp. 1361-1364.

[8] H. L. V. Trees, Detection, Estimation and Modulation Theory, Part 1. John Wiley Sons, 1968.

[9] R. Raheli, A. Polydoros, and C. Tzou, "Per-survivor processing: a general approach to MLSE in uncertain environments," IEEE Trans. Commun., vol. 43, pp. 354-364, 1995.

[10] I. S. Gradshteyn and I. M. Ryzhik, Table of Integrals, Series, and Products, 6th edition. Academic, 2000.

[11] S. G. Wilson, M. Brandt-Pearce, C. Qianling, and M. Baedke, "Optical repetition MIMO transmission with multipulse PPM," IEEE J. Sel. Areas Commun., vol. 23, no. 9, pp. 1901-1910, 2005.

[12] S. M. Navidpour and M. Uysal, "BER performance of free-space optical transmission with spatial diversity," IEEE Trans. Wireless Commun., vol. 6, no. 8, pp. 2813-2819, Aug. 2007.

[13] D. J. T. Heatley, D. R. Wisely, I. Neild, and P. Cochrane, "Optical wireless: the story so far," IEEE Commun. Mag., vol. 36, no. 2, pp. 72-74, Dec. 1998.

[14] E. Lee and V. Chan, "Part 1: optical communication over the clear turbulent atmospheric channel using diversity," IEEE J. Sel. Areas Commun., vol. 22, no. 9, pp. 71 896-1906, Nov. 2004.

[15] R. Gagliardi and S. Karp, Optical Communications, 1st edition. Wiley \& Sons, Inc., 1976. 